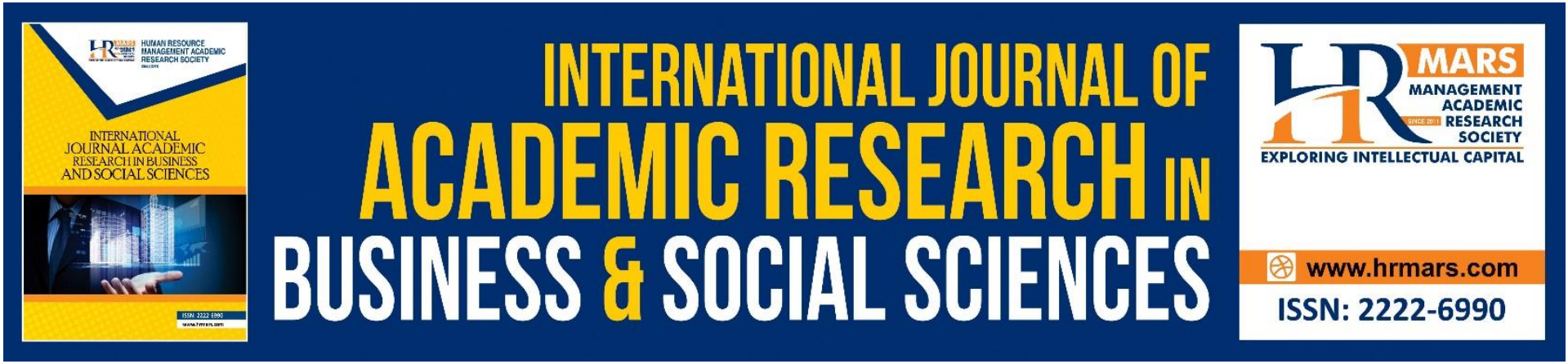

\title{
Integration of the History of Mathematics in Mathematics Education: A Systematic Literature Review
}

\author{
Nur Amalia Azman, Siti Mistima Maat
}

To Link this Article: http://dx.doi.org/10.6007/IJARBSS/v11-i4/9768

DOI:10.6007/IJARBSS/v11-i4/9768

Received: 04 February 2021, Revised: 10 March 2021, Accepted: 28 March 2021

Published Online: 25 April 2021

In-Text Citation: (Azman \& Maat, 2021)

To Cite this Article: Azman, N. A., \& Maat, S. M. (2021). Integration of the History of Mathematics in Mathematics Education: A Systematic Literature Review. International Journal of Academic Research in Business and Social Sciences, 11(4), 1035-1057.

Copyright: @ 2021 The Author(s)

Published by Human Resource Management Academic Research Society (www.hrmars.com) This article is published under the Creative Commons Attribution (CC BY 4.0) license. Anyone may reproduce, distribute, translate and create derivative works of this article (for both commercial and non-commercial purposes), subject to full attribution to the original publication and authors. The full terms of this license may be seen at: http://creativecommons.org/licences/by/4.0/legalcode

Vol. 11, No. 4, 2021, Pg. 1035 - 1057

Full Terms \& Conditions of access and use can be found at http://hrmars.com/index.php/pages/detail/publication-ethics 


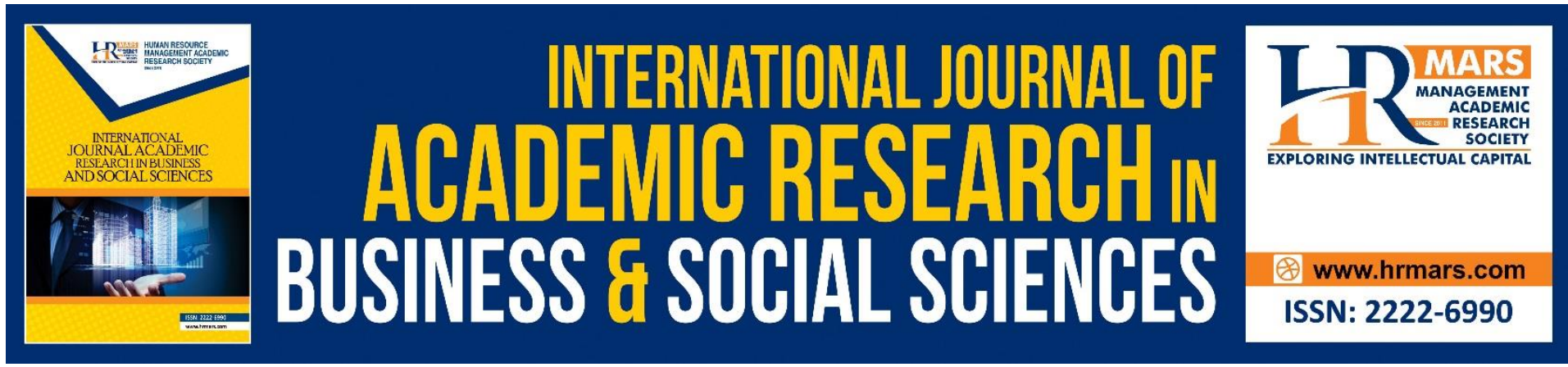

\title{
Integration of the History of Mathematics in Mathematics Education: A Systematic Literature Review
}

\author{
Nur Amalia Azman, Siti Mistima Maat \\ Faculty of Education, Universiti Kebangsaan Malaysia, Bangi, Selangor, Malaysia \\ Email: sitimistima@ukm.edu.my
}

\begin{abstract}
The history of mathematics (HoM) is important for mathematics to be enriched, in the evaluation of the development of the mathematics based on scholars and subjects. However, many students often have a bad impression of Mathematics. Therefore, a lot of alternatives have been used by Mathematics educators to change this sceptical view of Mathematics. One of the methods is by using the history in Mathematics in the teaching and learning process. Therefore, this study aims to identify Mathematics teachers' views towards the integration of the HoM and the implementation of HoM in teaching and learning in a systematic literature review context. Using the guidelines by Preferred Reporting Items for Systematic Review and Meta-Analyzes (PRISMA) and the combination of keywords such as "integration of History of Mathematics", "using History of Mathematics", "implementing History of Mathematics" and "integrating History of Mathematics". 30 out of 1412 articles used in this study were chosen from two electronic databases which are ERIC, and Google Scholar without restricting the year of publication. The article selection was done based on a few criteria such that the variables in previous research were focused on the views and opinions of teachers about the usage of $\mathrm{HoM}$ and the usage of HoM in Mathematics education. Extraction of data was based on the name of the researcher, year, country, title, sample size, instrument used, field of study, methodology and analysis and findings. The findings reveal that Mathematics' teachers have positive views about the integration of HoM in teaching and learning. Teachers did not use HoM in class due to problems such as lack of knowledge and skill to integrate HoM, insufficient materials, resources and time and exclusion of HoM in the Mathematics syllabus and examination. To conclude, Mathematics teachers should consider integrating HoM in their teaching and choosing history as an alternative way to teach Mathematics. As for future works, Mendeley, Scopus and Web of Science are highly recommended to be databases of the study using should extensive related keywords.
\end{abstract}

Keywords: Integration, History of Mathematics, Systematic Literature Review.

\section{Introduction}

Mathematics is a very important discipline in everyday life because it is a field that all people need to use and practice in everyday life (Bailey, 2017). However, Mathematics is 
often labeled as a subject that is feared by most students not only at school level, but also among higher education students. Today, Mathematics anxiety is affecting most students because they think that Mathematics is difficult to learn because they cannot understand and master the concepts presented in this subject (Nachiappan et al., 2016). In addition, almost all of the concepts and elements learned in this subject are abstract and therefore students have difficulty in integrating them into their real lives (Bailey, 2017). In Malaysia, local research done by Hong Choon and Chandrasekaran (2015) found that every time a new mathematical symbol was introduced in Mathematics it had a negative impact on students' mathematics achievements. Despite this, some local Mathematics teachers came from nonmathematics background with a low level of content knowledge and this indirectly influenced their teaching performances as well as students' interest in learning Mathematics (Li et al., 2019). As a result, these factors cause students to have an idea that Mathematics is a hard, boring and unpleasant subject in school and that leaves them with negative attitudes towards it.

As such, various initiatives have been introduced by Mathematics teachers in order to help students who are experiencing phobias towards Mathematics to destroy and eliminate their fears and negative perceptions of this subject. One of the exciting initiatives that has begun to attract teachers' attention to enhance and diversify the teaching and learning of Mathematics in the classroom is by integrating the History of Mathematics (HoM) into Mathematics teaching. HoM is a scientific field which is known as one of the most crucial branches of Mathematical knowledge. This field describes the journey and development of Mathematics as well as the Mathematicians involved in the formulation of concepts as well as developing Mathematical formulas (Baki \& Gürsoy, 2018).

Teachers choose to use HoM during their teaching sessions to develop affective and cognitive skills as well as to help students in understanding the nature of Mathematics. In addition, HoM is also used to ensure students' engagement in the classroom, gain their attention, arouse curiosity and help them to construct positive beliefs and attitudes towards Mathematics. HoM can also help students to understand the origins of a topic in Mathematics in addition to helping them to relate what they have learned in Mathematics to their everyday life (Bașibüyük \& Șahin, 2019). Bütüner and Baki (2020) stated that students' beliefs about Mathematics can be increased by applying HoM in Mathematics classes through fun, useful and interesting class activities. In addition, the usage of HoM also helps students to develop meaningful relationships between abstract mathematical ideas and practical applications in real-world contexts (Bailey, 2017). It is believed that the learning environment plays an important role in helping students to develop thinking skills in Mathematics (Hamidah et al., 2019). Therefore, the usage of HoM in teaching can be considered a brilliant step to help students in learning Mathematics, enrich teaching content and create an exciting learning environment. Moreover, the integration of HoM in the classroom has been widely supported by many researchers (Astin et al., 2016; Bidwell, 1993; Fauvel, 1991; Fried, 2001; Gulikers \& Blom, 2001; Jankvist, 2009; Liu, 2003; Tzanakis et al., 2002). Several groups such as the National Council of Teachers of Mathematics (NCTM) and The Mathematical Association of America (MAA) have also proposed and recommended the integration of HoM in the classroom (Baki \& Guven, 2009).

In a study conducted by Tzanakis et al. (2002), they highlighted several ways that teachers can integrate HoM in the teaching and learning process. These include (1) usage of historical quotes in teaching, (2) conducting research projects involving the use of historical sources, (3) usage of original sources for a topic, (4) incorporating HoM elements in practising 
of mathematics questions (5) discussing problems arising from the discovery of Mathematics based on its history, (6) use of mechanical materials, (7) conducting experimental-based Mathematical activities, (8) presenting HoM in the form of games, (9) using film, video or other visual materials, (10) emphasising external experiences and (11) encouraging the search for the origin of a mathematical concept through the internet.

In addition, most studies have found that the usage of HoM in teaching Mathematics has many positive effects on teachers, students and the process of teaching and learning Mathematics itself (Hickman \& Kapadia, 1983; Philippou \& Christou, 1998; Marshall \& Rich, 2000; Liu, 2003; Charalambous, Panaoura, \& Philippou, 2009). HoM is also seen as a medium that can serve as a guide for developing mathematical instruction designs that include a variety of aspects such as teaching strategies, problem solving and interesting topics that can be used for classroom discussion (Galante, 2014). Therefore, this systematic literature review aims to review articles on integration of HoM in Mathematics education. The objectives of this study are as follows:

- To identify Mathematics teachers' views on the integration of HoM in Mathematics teaching and learning sessions.

- To investigate the implementation of HoM in teaching and learning Mathematics.

\section{Methodology of Research}

\section{General Background of the Research}

This study uses a systematic literature review method. According to Cooper (2003), systematic review is a method for determining, assessing critically and deciphering research discoveries identified with research questions, topics or the significance of such phenomena. Therefore, this is the best method to study related articles on the integration of HoM. Thirty articles were involved in this study on Mathematics teachers' views and opinions about the integration of HoM in the classroom including the practice of the integration of HoM in teaching and learning processes as well as the benefits of it. A qualitative analysis method has been used in this study by conducting content analysis to analyse the results for this study. The guidelines introduced by Moher, Liberati, and Tetzlaff, (2009) in Preferred Reporting Items for Systematic Reviews and Meta-Analyses (PRISMA) is followed in order to handle and report the outcomes of this study.

\section{Selection Criteria}

This study only considered articles generated from article writing on the integration of HoM in Mathematics education without restricting the year of publication. Sources of writing such as blogging and unpublished reports were excluded in this study because these types of sources do not go through the peer review process. Thus, the credibility and objectivity of the writing should be taken into account (Liyanagunawardena et al. 2013). The selected articles are either qualitative or quantitative and involve only open-access articles to ease the downloading process for the full-text articles. This study also involved articles which are written in English only.

\section{Searching Strategy}

The articles involved in this study were chosen by focusing on the integration of HoM in Mathematics education and published at any time. Two electronic databases, the Education Resources Information Center (ERIC) and Google Scholar, were used to find the related articles. These databases were chosen because Google Scholar is a database that has 
accumulated articles from different databases while ERIC is a database that can be used to find educational related articles. The combination of keywords that was used to conduct searches in the databases listed was: "integration of History of Mathematics", "using History of Mathematics", "implementing History of Mathematics" and "integrating History of Mathematics". The articles and abstracts went through preliminary checks before finding the full text for the articles that met the criteria. Final selection of articles was determined by selecting only the articles that satisfied the needed criteria.

\section{Data Extraction}

For the preliminary check, the researcher went through the title of the article and abstract to select suitable articles. Articles that did not satisfy the criteria needed were excluded. Then, the researcher downloaded the full text of the remaining articles and read all of them to determine which articles needed to be included or excluded in this study. The researcher then constructed a table for data extraction including the name of the researcher(s), publication year, publication country, title, sample size, instrument used, variable, methodology and analysis and lastly the research findings.

\section{Results of Research}

This paper aims to study articles relating to the integration of the History of Mathematics (HoM). This systematic survey explores Mathematics teachers' views about the integration of HoM in Mathematics education and the usage of HoM in Mathematics education. The flow of the article selection is shown in Figure 1. 


\section{Figure 1: The flowchart of article selection}

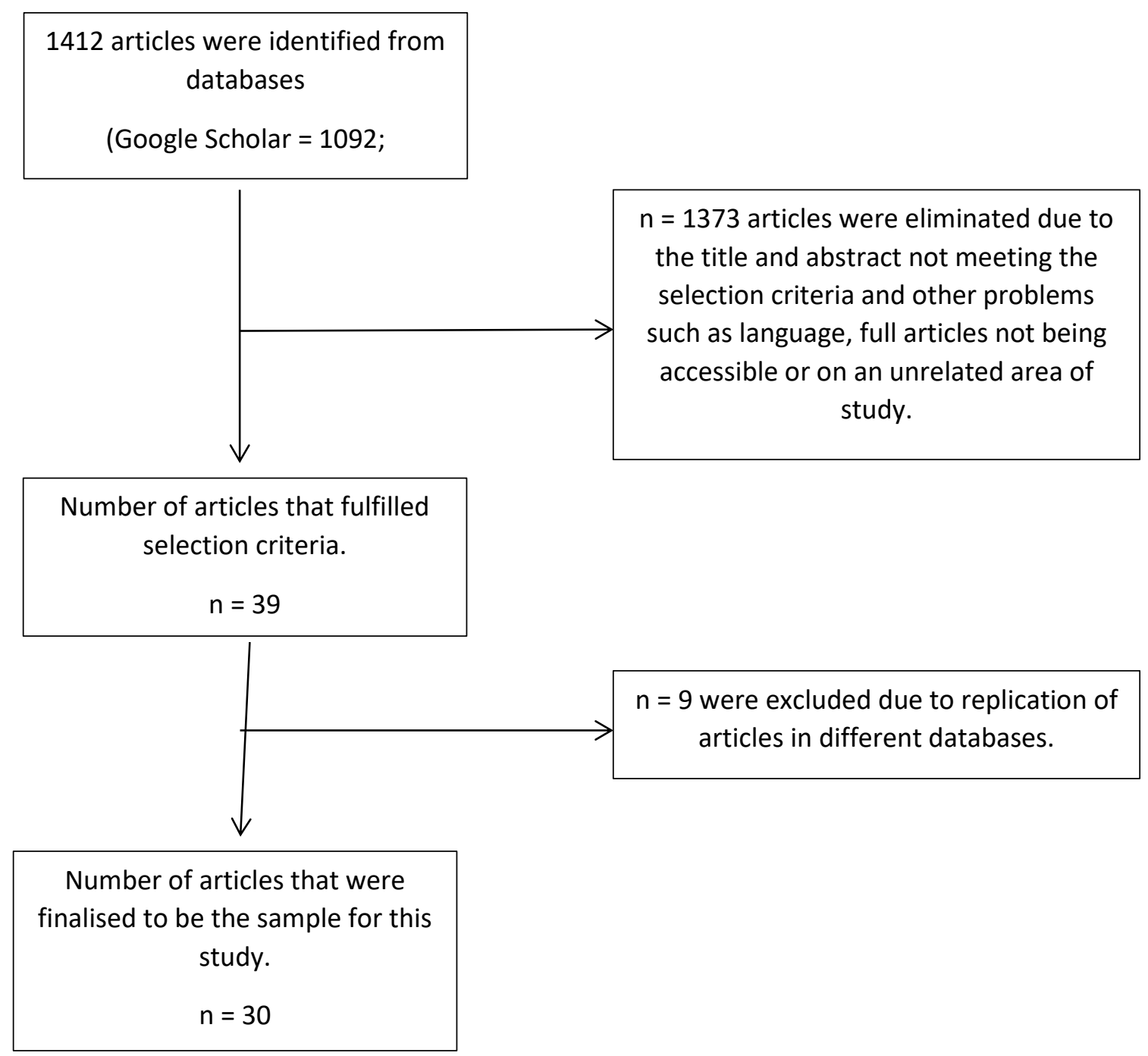

Initially, 1412 articles were identified by the search protocol. Overall, 1373 were then eliminated after going through an inspection by title and abstract. Most of the articles were eliminated due to the different areas of study related to HoM and different characteristics of the sample studied. Next, 39 articles were left. From the articles left, 9 were eliminated due to replication of articles in different databases. Finally, 30 articles were included in this systematic review study.

Table 1 below shows a summary of the articles involved in this systematic survey. From 30 articles that matched the selection criteria for this survey, 12 were done in Turkey, 5 in the United States, 2 in Indonesia, 2 in Singapore, 1 respectively in Ethiopia, Greece, Germany, Taiwan, Denmark, Serbia, South Africa, China and Australia. Fifteen of the selected articles studied were on the views of Mathematics' teacher about HoM while 19 articles studied were on the integration of HoM in Mathematics education. The methods used to study the opinions about HoM were: test, questionnaire, interview, survey and observation and also reflective writing. Meanwhile, the methods used to study the practice of HoM were: knowledge test, written opinion form, reflective journals, constant comparative method and case study. 
INTERNATIONAL JOURNAL OF ACADEMIC RESEARCH IN BUSINESS AND SOCIAL SCIENCES Vol. 11, No. 4, 2021, E-ISSN: 2222-6990 @ 2021 HRMARS

\section{Table 1. Summary of selected articles}

\begin{tabular}{|c|c|c|c|c|c|c|c|c|}
\hline No & $\begin{array}{l}\text { Researc } \\
\text { her (s) }\end{array}$ & $\begin{array}{l}\text { Countr } \\
y\end{array}$ & Title & Population & Instrument & $\begin{array}{l}\text { Field of } \\
\text { study }\end{array}$ & $\begin{array}{l}\text { Methodolo } \\
\text { gy/ Analysis }\end{array}$ & Findings \\
\hline 1 & $\begin{array}{l}\text { Fraser } \\
\& \text { Koop } \\
(1978)\end{array}$ & $\begin{array}{l}\text { Australi } \\
\mathrm{a}\end{array}$ & $\begin{array}{l}\text { Teachers' } \\
\text { opinions about } \\
\text { some teaching } \\
\text { material } \\
\text { involving the } \\
\text { history of } \\
\text { mathematics }\end{array}$ & $\begin{array}{l}39 \\
\text { mathematic } \\
\text { s teachers }\end{array}$ & Questionnaires & $\begin{array}{l}\text { Views } \\
\text { about } \\
\text { HoM }\end{array}$ & T-test & $\begin{array}{l}\text { Teachers' have positive impressions } \\
\text { about using HoM materials but they } \\
\text { did not consider using it in teaching } \\
\text { because of a lack of time and skills }\end{array}$ \\
\hline 2 & $\begin{array}{l}\text { Tzanaki } \\
\text { s et al. } \\
(2002)\end{array}$ & Greece & $\begin{array}{l}\text { Integrating } \\
\text { history of } \\
\text { mathematics in } \\
\text { the classroom: } \\
\text { an analytic } \\
\text { survey }\end{array}$ & - & Analytic survey & $\begin{array}{l}\text { Integratio } \\
\mathrm{n} \text { of HoM }\end{array}$ & $\begin{array}{l}\text { Content } \\
\text { analysis }\end{array}$ & $\begin{array}{l}\text { Ways to integrate HoM in class: give } \\
\text { direct information, use historical- } \\
\text { inspired teaching strategy, spread } \\
\text { awareness in social and cultural } \\
\text { contexts of Mathematics. } \\
\text { Examples of tools to integrate HoM in } \\
\text { class: historical snippets, history- } \\
\text { based projects, primary sources, } \\
\text { worksheets, historical packages, } \\
\text { discussion on historical issues and } \\
\text { arguments, historical problems, } \\
\text { mechanical instruments, experiential } \\
\text { activities, plays, visual tools, outdoor } \\
\text { learning and internet sources. }\end{array}$ \\
\hline 3 & $\begin{array}{l}\text { Liu } \\
\text { (2003) }\end{array}$ & Taiwan & $\begin{array}{lr}\text { Do Teachers } \\
\text { Need ro } \\
\text { Incorporate the } \\
\text { History of }\end{array}$ & - & $\begin{array}{l}\text { Content } \\
\text { analysis }\end{array}$ & $\begin{array}{l}\text { Integratio } \\
\mathrm{n} \text { of HoM }\end{array}$ & $\begin{array}{l}\text { Literature } \\
\text { review }\end{array}$ & $\begin{array}{l}\text { Reasons to integrate HoM in } \\
\text { teaching: develop positive attitudes } \\
\text { and increase motivation towards } \\
\text { learning Mathematics, help to } \\
\text { explain difficulties faced by students }\end{array}$ \\
\hline
\end{tabular}


INTERNATIONAL JOURNAL OF ACADEMIC RESEARCH IN BUSINESS AND SOCIAL SCIENCES Vol. 11, No. 4, 2021, E-ISSN: 2222-6990 @ 2021 HRMARS

\begin{tabular}{|c|c|c|c|c|c|c|c|c|}
\hline & & & $\begin{array}{l}\text { Mathematics in } \\
\text { their Teaching? }\end{array}$ & & & & & $\begin{array}{l}\text { in problem solving, trigger students' } \\
\text { mathematical thinking and explain } \\
\text { human side of mathematical } \\
\text { knowledge. }\end{array}$ \\
\hline 4 & $\begin{array}{l}\text { Ho } \\
\text { (2008) }\end{array}$ & $\begin{array}{l}\text { Singapo } \\
\text { re }\end{array}$ & $\begin{array}{l}\text { Using history of } \\
\text { mathematics in } \\
\text { the teaching and } \\
\text { learning of } \\
\text { mathematics in } \\
\text { Singapore }\end{array}$ & $\begin{array}{l}102 \\
\text { students }\end{array}$ & $\begin{array}{l}\text { 1. Record of } \\
\text { the teaching } \\
\text { and learning } \\
\text { process } \\
2 . \quad \text { Student } \\
\text { survey }\end{array}$ & $\begin{array}{l}\text { Integratio } \\
\mathrm{n} \text { of HoM }\end{array}$ & $\begin{array}{l}1 . \quad \text { Case } \\
\text { study } \\
2 . \\
\text { Qualitative } \\
\text { analysis }\end{array}$ & $\begin{array}{l}\text { Benefits of integrating HoM: better } \\
\text { understanding, creating different } \\
\text { learning environment and } \\
\text { developing positive attitudes } \\
\text { towards teaching and learning } \\
\text { Mathematics. } \\
\text { Limitation: lack of teachers training, } \\
\text { time and rubrics } \\
\text { Risks of using HoM: cannot make a } \\
\text { relevant connection between HoM } \\
\text { and the topic, time issues and wrong } \\
\text { interpretation }\end{array}$ \\
\hline 5 & $\begin{array}{l}\text { Jankvist } \\
\text { (2009) }\end{array}$ & $\begin{array}{l}\text { Denma } \\
\text { rk }\end{array}$ & $\begin{array}{l}\text { A categorisation } \\
\text { of the whys and } \\
\text { hows of using } \\
\text { history in } \\
\text { mathematics } \\
\text { education. }\end{array}$ & - & $\begin{array}{l}\text { Content } \\
\text { analysis }\end{array}$ & $\begin{array}{l}\text { Integratio } \\
n \text { of HoM }\end{array}$ & $\begin{array}{l}\text { Literature } \\
\text { review }\end{array}$ & $\begin{array}{l}\text { Usage of HoM: teaching and learning } \\
\text { tools, as a goal in development of } \\
\text { Mathematics as a discipline. } \\
\text { How to use HoM in education: } \\
\text { Illumination approach, Modules } \\
\text { approach and History-based } \\
\text { approach }\end{array}$ \\
\hline 6 & $\begin{array}{l}\text { Burns } \\
\text { (2010) }\end{array}$ & $\begin{array}{l}\text { United } \\
\text { States }\end{array}$ & $\begin{array}{l}\text { Pre-Service } \\
\text { Teachers' } \\
\text { Exposure to } \\
\text { Using the } \\
\text { History of } \\
\text { Mathematics to } \\
\text { Enhance Their }\end{array}$ & $\begin{array}{l}32 \text { pre- } \\
\text { service } \\
\text { teachers }\end{array}$ & $\begin{array}{l}\text { Questionnaire, } \\
\text { open-ended } \\
\text { question and } \\
\text { final reflection } \\
\text { paper }\end{array}$ & $\begin{array}{l}\text { Views } \\
\text { about } \\
\text { HoM }\end{array}$ & $\begin{array}{l}\text { Paired } \\
\text { sample t- } \\
\text { test } \\
\text { Grounded } \\
\text { theory } \\
\text { approach }\end{array}$ & $\begin{array}{l}\text { Pre-service teachers have positive } \\
\text { views about integrating the HoM } \\
\text { after being exposed in terms of how } \\
\text { to use HoM. }\end{array}$ \\
\hline
\end{tabular}


INTERNATIONAL JOURNAL OF ACADEMIC RESEARCH IN BUSINESS AND SOCIAL SCIENCES Vol. 11, No. 4, 2021, E-ISSN: 2222-6990 @ 2021 HRMARS

\begin{tabular}{|c|c|c|c|c|c|c|c|c|}
\hline & & & $\begin{array}{l}\text { Teaching of High } \\
\text { School } \\
\text { Mathematics }\end{array}$ & & & & & \\
\hline 7 & $\begin{array}{l}\text { Bolinge } \\
r- \\
\text { Horton } \\
\& \\
\text { Panasu } \\
k \\
(2011)\end{array}$ & $\begin{array}{l}\text { United } \\
\text { States }\end{array}$ & $\begin{array}{l}\text { Raising } \\
\text { Awareness of } \\
\text { the History of } \\
\text { Mathematics in } \\
\text { High School } \\
\text { Curriculum }\end{array}$ & $\begin{array}{l}367 \\
\text { Mathematic } \\
\text { s teachers }\end{array}$ & Survey & $\begin{array}{l}\text { Integratio } \\
\mathrm{n} \text { of HoM }\end{array}$ & $\begin{array}{l}\text { Independen } \\
\mathrm{t} \text { sample } \mathrm{T} \text { - } \\
\text { test analysis }\end{array}$ & $\begin{array}{l}\text { Teachers who have a fallibilist } \\
\text { perspective towards the nature of } \\
\text { Mathematics are more likely to } \\
\text { include HoM in their lessons } \\
\text { compared to teachers who have an } \\
\text { absolutist perspective towards the } \\
\text { nature of Mathematics. Teachers } \\
\text { who have an absolutist perspective } \\
\text { have a fixed procedure in their } \\
\text { teaching process and are less } \\
\text { interested in creating a new learning } \\
\text { environment while teachers with a } \\
\text { fallibilist perspective are flexible to } \\
\text { change their teaching style. }\end{array}$ \\
\hline 8 & $\begin{array}{l}\text { Panasu } \\
k \quad \& \\
\text { Bolinge } \\
\text { r- } \\
\text { Horton } \\
(2011)\end{array}$ & $\begin{array}{l}\text { United } \\
\text { States }\end{array}$ & $\begin{array}{l}\text { Integrating } \\
\text { History of } \\
\text { Mathematics } \\
\text { into Curriculum: } \\
\text { What are the } \\
\text { Chances and } \\
\text { Constraints? }\end{array}$ & $\begin{array}{l}367 \\
\text { Mathematic } \\
\text { s teachers }\end{array}$ & $\begin{array}{l}\text { Online } \\
\text { comprehensiv } \\
\text { e survey }\end{array}$ & $\begin{array}{l}\text { 1. Views } \\
\text { about } \\
\text { HoM } \\
2 . \\
\text { Integratio } \\
\text { n of HoM }\end{array}$ & $\begin{array}{l}\text { Factor } \\
\text { analysis }\end{array}$ & $\begin{array}{l}\text { Most of the teachers have good } \\
\text { perceptions of HoM. They believed } \\
\text { that HoM has its value and place in } \\
\text { the school curriculum and would help } \\
\text { to enhance students' motivation and } \\
\text { interest in learning Mathematics. } \\
\text { However, some of the teachers } \\
\text { viewed HoM as burdening. } \\
\text { Factors that affect teachers' decision } \\
\text { in applying HoM are their beliefs } \\
\text { about the importance of HOM, } \\
\text { benefits of HoM and values of HoM. }\end{array}$ \\
\hline
\end{tabular}


INTERNATIONAL JOURNAL OF ACADEMIC RESEARCH IN BUSINESS AND SOCIAL SCIENCES Vol. 11, No. 4, 2021, E-ISSN: 2222-6990 @ 2021 HRMARS

\begin{tabular}{|c|c|c|c|c|c|c|c|c|}
\hline 9 & $\begin{array}{l}\text { Alpasla } \\
\mathrm{n} \quad \& \\
\text { Haser } \\
(2012)\end{array}$ & Turkey & $\begin{array}{l}\text { "History Of } \\
\text { Mathematics" } \\
\text { Course For Pre- } \\
\text { Service } \\
\text { Mathematics } \\
\text { Teachers: A Case } \\
\text { Study }\end{array}$ & $\begin{array}{l}24 \quad \text { pre- } \\
\text { service } \\
\text { mathematic } \\
\text { s teachers }\end{array}$ & $\begin{array}{l}\text { Observation of } \\
\text { the course } \\
\text { setting, } \\
\text { interviews }\end{array}$ & $\begin{array}{l}\text { Views } \\
\text { about } \\
\text { HoM }\end{array}$ & $\begin{array}{l}\text { Descriptive } \\
\text { analysis }\end{array}$ & $\begin{array}{l}\text { Pre-service teachers have positive } \\
\text { attitudes and beliefs about the usage } \\
\text { of HoM but did not consider that } \\
\text { learning about HoM is so important. }\end{array}$ \\
\hline 10 & $\begin{array}{l}\text { Ozdemi } \\
\text { r, } \\
\text { Goktep } \\
\text { e, \& } \\
\text { Kepceo } \\
\text { glu } \\
(2012)\end{array}$ & Turkey & $\begin{array}{l}\text { Using } \\
\text { mathematics } \\
\text { history to } \\
\text { strengthen } \\
\text { geometric proof } \\
\text { skills }\end{array}$ & $\begin{array}{l}15 \text { students } \\
\text { from } 11^{\text {th }} \\
\text { grade }\end{array}$ & Questionnaire & $\begin{array}{l}\text { Integratio } \\
\mathrm{n} \text { of HoM }\end{array}$ & $\begin{array}{l}\text { Multiple } \\
\text { case study }\end{array}$ & $\begin{array}{l}\text { Using HoM helped to strengthen } \\
\text { students' geometric proof skills } \\
\text { besides attracting their interest to } \\
\text { learn the topic. }\end{array}$ \\
\hline 11 & $\begin{array}{l}\text { Goktep } \\
\text { e \& } \\
\text { Ozdemi } \\
\text { r (2013) }\end{array}$ & Turkey & $\begin{array}{l}\text { An example of } \\
\text { using history of } \\
\text { mathematics in } \\
\text { classes }\end{array}$ & $\begin{array}{l}21 \text { students } \\
\text { in private } \\
\text { primary } \\
\text { school }\end{array}$ & $\begin{array}{l}\text { 1. Worksheets } \\
\text { 2. Interview }\end{array}$ & $\begin{array}{l}\text { Integratio } \\
\mathrm{n} \text { of HoM }\end{array}$ & $\begin{array}{l}\text { Multiple } \\
\text { case study }\end{array}$ & $\begin{array}{l}\text { Integration of HoM in classroom } \\
\text { teaching attracts students' interests } \\
\text { and helps them to discover different } \\
\text { methods to solve mathematical } \\
\text { problems. }\end{array}$ \\
\hline 12 & $\begin{array}{l}\text { Panasu } \\
\text { k \& } \\
\text { Horton } \\
(2013)\end{array}$ & $\begin{array}{l}\text { United } \\
\text { States }\end{array}$ & $\begin{array}{l}\text { Integrating } \\
\text { History of } \\
\text { Mathematics } \\
\text { into the } \\
\text { Classroom: Was } \\
\text { Aristotle } \\
\text { Wrong? }\end{array}$ & $\begin{array}{l}367 \\
\text { Mathematic } \\
\text { s teachers }\end{array}$ & $\begin{array}{l}\text { On-line } \\
\text { comprehensiv } \\
\text { e survey }\end{array}$ & $\begin{array}{l}\text { Integratio } \\
n \text { of HoM }\end{array}$ & $\begin{array}{l}\text { Descriptive } \\
\text { analysis }\end{array}$ & $\begin{array}{l}\text { Factors influencing usage of HOM: } \\
\text { teacher knowledge, resources, high } \\
\text { stakes testing and enjoyment in using } \\
\text { HOM. }\end{array}$ \\
\hline 13 & $\begin{array}{l}\text { Alpasla } \\
\text { n, } \\
\text { Işiksal, }\end{array}$ & Turkey & $\begin{array}{l}\text { Pre-service } \\
\text { Mathematics } \\
\text { Teachers' } \\
\text { Knowledge of }\end{array}$ & $\begin{array}{l}1593 \text { pre- } \\
\text { service } \\
\text { Mathematic } \\
s^{\prime} \text { teachers }\end{array}$ & $\begin{array}{l}\text { 1. Knowledge } \\
\text { of History of } \\
\text { Mathematics } \\
\text { Test }\end{array}$ & $\begin{array}{l}\text { Views } \\
\text { about } \\
\text { HoM }\end{array}$ & $\begin{array}{l}\text { 1. Cross- } \\
\text { sectional } \\
\text { survey }\end{array}$ & $\begin{array}{l}\text { Pre-service teachers have moderate } \\
\text { knowledge of HoM and positive } \\
\text { attitudes and beliefs about using } \\
\text { HoM. }\end{array}$ \\
\hline
\end{tabular}


INTERNATIONAL JOURNAL OF ACADEMIC RESEARCH IN BUSINESS AND SOCIAL SCIENCES Vol. 11, No. 4, 2021, E-ISSN: 2222-6990 @ 2021 HRMARS

\begin{tabular}{|c|c|c|c|c|c|c|c|c|}
\hline & $\begin{array}{l}\text { \& Haser } \\
(2014)\end{array}$ & & $\begin{array}{l}\text { History of } \\
\text { Mathematics } \\
\text { and Their } \\
\text { Attitudes and } \\
\text { Beliefs Towards } \\
\text { Using History of } \\
\text { Mathematics in } \\
\text { Mathematics } \\
\text { Education }\end{array}$ & & $\begin{array}{l}2 . \quad \text { Attitudes } \\
\text { and Beliefs } \\
\text { about the Use } \\
\text { of History of } \\
\text { Mathematics } \\
\text { in } \\
\text { Mathematics } \\
\text { Education } \\
\text { Questionnaire }\end{array}$ & & $\begin{array}{l}\text { research } \\
\text { design } \\
2 . \\
\text { Descriptive } \\
\text { statistics } \\
3 \text {. Two-way } \\
\text { Analysis of } \\
\text { Variance }\end{array}$ & \\
\hline 14 & $\begin{array}{l}\text { Dejic \& } \\
\text { Mihajlo } \\
\text { vic } \\
(2014)\end{array}$ & Serbia & $\begin{array}{l}\text { History of } \\
\text { Mathematics } \\
\text { and Teaching } \\
\text { Mathematics }\end{array}$ & $\begin{array}{l}112 \\
\text { teachers }\end{array}$ & Survey & $\begin{array}{l}1 . \quad \text { Views } \\
\text { about } \\
\text { HoM } \\
2 . \\
\text { Integratio } \\
\text { n of HoM }\end{array}$ & $\begin{array}{l}\text { Quantitativ } \\
\text { e analysis }\end{array}$ & $\begin{array}{l}\text { Teachers have positive perceptions } \\
\text { about HoM but they did not integrate } \\
\text { HoM in their teaching due to lack of } \\
\text { resources, methodology and time. }\end{array}$ \\
\hline 15 & $\begin{array}{l}\text { Galant } \\
(2014)\end{array}$ & $\begin{array}{l}\text { United } \\
\text { States }\end{array}$ & $\begin{array}{l}\text { The Use of the } \\
\text { History of } \\
\text { Mathematics in } \\
\text { the Teaching of } \\
\text { Preservice } \\
\text { Mathematics } \\
\text { Teachers }\end{array}$ & $\begin{array}{l}9 \quad \text { pre- } \\
\text { service } \\
\text { teachers }\end{array}$ & $\begin{array}{l}\text { 1. Reflective } \\
\text { journals } \\
\text { written by } \\
\text { participants } \\
\text { 2. Constant } \\
\text { comparative } \\
\text { method } \\
\text { 3. Case study }\end{array}$ & $\begin{array}{l}\text { Views } \\
\text { about } \\
\text { HoM }\end{array}$ & $\begin{array}{l}1 . \\
\text { Grounded } \\
\text { theory } \\
\text { approach } \\
2 . \\
\text { Qualitative } \\
\text { analysis }\end{array}$ & $\begin{array}{l}\text { Pre-service teachers believed usage } \\
\text { of HoM in teaching is beneficial in the } \\
\text { context of content knowledge and } \\
\text { pedagogical content knowledge. }\end{array}$ \\
\hline 16 & $\begin{array}{l}\text { Weldea } \\
\text { na \& } \\
\text { Abraha } \\
\text { m } \\
(2014)\end{array}$ & $\begin{array}{l}\text { Ethiopi } \\
\text { a }\end{array}$ & $\begin{array}{l}\text { The effect of an } \\
\text { historical } \\
\text { perspective on } \\
\text { prospective } \\
\text { teachers' beliefs } \\
\text { in learning } \\
\text { mathematics }\end{array}$ & $\begin{array}{l}63 \text { pre- } \\
\text { service } \\
\text { teachers }\end{array}$ & $\begin{array}{l}\text { Questionnaire } \\
\text { Reflective } \\
\text { writing }\end{array}$ & $\begin{array}{l}\text { Views } \\
\text { about } \\
\text { HoM }\end{array}$ & $\begin{array}{l}\text { T-test } \\
\text { Kitwood's } \\
\text { method of } \\
\text { analysing } \\
\text { accounts }\end{array}$ & $\begin{array}{l}\text { History-based intervention provides } \\
\text { a positive impact in shifting } \\
\text { prospective teachers' beliefs to } \\
\text { progressive beliefs. }\end{array}$ \\
\hline
\end{tabular}


INTERNATIONAL JOURNAL OF ACADEMIC RESEARCH IN BUSINESS AND SOCIAL SCIENCES Vol. 11, No. 4, 2021, E-ISSN: 2222-6990 @ 2021 HRMARS

\begin{tabular}{|c|c|c|c|c|c|c|c|c|}
\hline 17 & $\begin{array}{l}\text { Bütüne } \\
\text { r (2015) }\end{array}$ & Turkey & $\begin{array}{l}\text { Impact of Using } \\
\text { History of } \\
\text { Mathematics on } \\
\text { Students' } \\
\text { Mathematics } \\
\text { Attitude: A } \\
\text { Meta-Analysis } \\
\text { Study }\end{array}$ & 6 studies & $\begin{array}{l}\text { Content } \\
\text { analysis }\end{array}$ & $\begin{array}{l}\text { Integratio } \\
\mathrm{n} \text { of HoM }\end{array}$ & $\begin{array}{l}\text { Meta- } \\
\text { analysis } \\
\text { method }\end{array}$ & 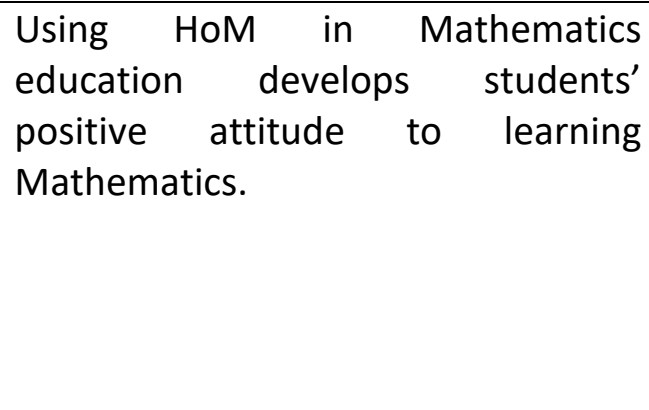 \\
\hline 18 & $\begin{array}{l}\text { Fadlelm } \\
\text { ula } \\
(2015)\end{array}$ & Turkey & $\begin{array}{l}\text { Pre-Service } \\
\text { Teachers' Point } \\
\text { Of Views About } \\
\text { Learning History } \\
\text { of Mathematics: } \\
\text { A Case Study In } \\
\text { Turkey }\end{array}$ & $\begin{array}{l}120 \text { pre- } \\
\text { service } \\
\text { teachers }\end{array}$ & $\begin{array}{l}\text { Open-ended } \\
\text { questionnaire }\end{array}$ & $\begin{array}{l}\text { Views } \\
\text { about } \\
\text { HoM }\end{array}$ & $\begin{array}{l}\text { Descriptive } \\
\text { statistics }\end{array}$ & $\begin{array}{l}\text { Pre-service teachers had positive } \\
\text { attitudes towards learning HoM. } \\
\text { They believed HoM is beneficial for } \\
\text { both personal and pedagogical } \\
\text { aspects. However, they had limited } \\
\text { ideas on how to implement HoM in } \\
\text { teaching. }\end{array}$ \\
\hline 19 & $\begin{array}{l}\text { Lim \& } \\
\text { Chapm } \\
\text { an } \\
(2015)\end{array}$ & $\begin{array}{l}\text { Singapo } \\
\text { re }\end{array}$ & $\begin{array}{l}\text { Effects of using } \\
\text { history as a tool } \\
\text { to teach } \\
\text { mathematics on } \\
\text { students' } \\
\text { attitudes, } \\
\text { anxiety, } \\
\text { motivation and } \\
\text { achievement in } \\
\text { grade } 11 \\
\text { classrooms }\end{array}$ & $\begin{array}{l}103 \\
\text { students }\end{array}$ & Tests & $\begin{array}{l}\text { Integratio } \\
\mathrm{n} \text { of HoM }\end{array}$ & $\begin{array}{l}\text { 1. Quasi- } \\
\text { experiment } \\
2 . \\
\text { Qualitative } \\
\text { feedback }\end{array}$ & $\begin{array}{l}\text { Implementing HoM in the class } \\
\text { provides both immediate short-term } \\
\text { and long-term effects on students' } \\
\text { achievement in Mathematics. } \\
\text { However, implementation of HoM } \\
\text { only has a short-term positive effects } \\
\text { on students' attitudes, anxiety and } \\
\text { motivation towards Mathematics. }\end{array}$ \\
\hline 20 & $\begin{array}{l}\text { Tol } \\
\text { (2015) }\end{array}$ & Turkey & $\begin{array}{lr}\text { Teachers' } & \text { Views } \\
\text { Related } & \text { To } \\
\text { Teaching Of } \\
\text { Mathematics }\end{array}$ & $\begin{array}{l}20 \\
\text { Mathematic } \\
\text { s teachers }\end{array}$ & $\begin{array}{l}\text { Open-ended } \\
\text { questions }\end{array}$ & $\begin{array}{l}\text { Views } \\
\text { about } \\
\text { HoM }\end{array}$ & $\begin{array}{l}\text { Descriptive } \\
\text { statistics } \\
\text { and content } \\
\text { analysis }\end{array}$ & $\begin{array}{l}\text { Teaching using HoM is more effective } \\
\text { to ensure permanent learning. } \\
\text { Teachers agreed with the idea of }\end{array}$ \\
\hline
\end{tabular}


INTERNATIONAL JOURNAL OF ACADEMIC RESEARCH IN BUSINESS AND SOCIAL SCIENCES Vol. 11, No. 4, 2021, E-ISSN: 2222-6990 @ 2021 HRMARS

\begin{tabular}{|c|c|c|c|c|c|c|c|c|}
\hline & & & $\begin{array}{l}\text { Course Subjects } \\
\text { With Their } \\
\text { Historical } \\
\text { Developments }\end{array}$ & & & & & $\begin{array}{l}\text { using HoM in class but rarely use it } \\
\text { due to not having sufficient material. }\end{array}$ \\
\hline 21 & $\begin{array}{l}\text { Bütüne } \\
\text { r(2017) }\end{array}$ & Turkey & $\begin{array}{l}\text { Secondary } \\
\text { School } \\
\text { Mathematics } \\
\text { Teachers' } \\
\text { Knowledge } \\
\text { Levels and Use } \\
\text { of History of } \\
\text { Mathematics }\end{array}$ & $\begin{array}{l}32 \\
\text { Mathematic } \\
\text { s teachers }\end{array}$ & $\begin{array}{l}1 . \quad 11 \text {-item } \\
\text { short-answer } \\
\text { HoM } \\
\text { knowledge } \\
\text { level test } \\
2 . \quad 5 \text {-item } \\
\text { written } \\
\text { opinion form }\end{array}$ & $\begin{array}{l}\text { Integratio } \\
\mathrm{n} \text { of HoM }\end{array}$ & $\begin{array}{l}\text { Descriptive } \\
\text { analysis }\end{array}$ & $\begin{array}{l}\text { Most teachers did not use HoM in } \\
\text { their classes because they feel } \\
\text { incompetent and had low HoM } \\
\text { knowledge levels. }\end{array}$ \\
\hline 22 & $\begin{array}{l}\text { Baki \& } \\
\text { Gürsoy } \\
\text { (2018) }\end{array}$ & Turkey & $\begin{array}{l}\text { Does Using } \\
\text { History of } \\
\text { Mathematics } \\
\text { Make Sense? } \\
\text { The Views of } \\
\text { Teacher } \\
\text { Candidates }\end{array}$ & $\begin{array}{l}6 \text { teacher } \\
\text { candidates }\end{array}$ & $\begin{array}{l}\text { Semi- } \\
\text { structured } \\
\text { interview }\end{array}$ & $\begin{array}{l}\text { Views } \\
\text { about } \\
\text { HoM }\end{array}$ & $\begin{array}{l}\text { Qualitative } \\
\text { analysis }\end{array}$ & $\begin{array}{l}\text { Teacher candidates believe that } \\
\text { using HoM in the instruction of } \\
\text { Mathematics may benefit both } \\
\text { teaching and learning purposes. } \\
\text { However, they have an issue } \\
\text { practicing this because of lack of } \\
\text { resources and time }\end{array}$ \\
\hline 23 & $\begin{array}{l}\text { Şişman } \\
\text { (2018) }\end{array}$ & Turkey & $\begin{array}{l}\text { History of } \\
\text { Mathematics in } \\
\text { the Turkish } \\
\text { Middle School } \\
\text { Mathematics } \\
\text { Curriculum and } \\
\text { Textbooks }\end{array}$ & $\begin{array}{l}\text { Turkish } \\
\text { middle } \\
\text { school } \\
\text { mathematic } \\
\text { s curriculum } \\
\text { (5th-8th } \\
\text { grades) and } \\
\text { textbooks }\end{array}$ & $\begin{array}{l}\text { Curriculum and } \\
\text { textbooks }\end{array}$ & $\begin{array}{l}\text { Integratio } \\
\mathrm{n} \text { of HoM }\end{array}$ & $\begin{array}{l}\text { Document } \\
\text { analysis }\end{array}$ & $\begin{array}{l}\text { Most of the HoM elements found in } \\
\text { the Mathematics textbooks were } \\
\text { placed in the introduction part of the } \\
\text { topic. HoM also was excluded from } \\
\text { the content and assessment aspects } \\
\text { of the curriculum. It can be } \\
\text { concluded that HoM is not being } \\
\text { integrated widely in the school } \\
\text { Mathematics curriculum. }\end{array}$ \\
\hline
\end{tabular}


INTERNATIONAL JOURNAL OF ACADEMIC RESEARCH IN BUSINESS AND SOCIAL SCIENCES Vol. 11, No. 4, 2021, E-ISSN: 2222-6990 @ 2021 HRMARS

\begin{tabular}{|c|c|c|c|c|c|c|c|c|}
\hline 24 & $\begin{array}{l}\text { Bașibüy } \\
\text { ük \& } \\
\text { Șahin } \\
\text { (2019) }\end{array}$ & Turkey & $\begin{array}{l}\text { Mathematics } \\
\text { Teachers' } \\
\text { Opinion About } \\
\text { The History of } \\
\text { Mathematics }\end{array}$ & $\begin{array}{l}21 \\
\text { Mathematic } \\
\text { s teachers }\end{array}$ & $\begin{array}{l}\text { Structured } \\
\text { interview form }\end{array}$ & $\begin{array}{l}\text { 1. Views } \\
\text { about } \\
\text { HoM } \\
2 . \\
\text { Integratio } \\
\text { n of HoM }\end{array}$ & $\begin{array}{l}1 . \\
\text { Qualitative } \\
\text { method of } \\
\text { case study } \\
2 \text {. Content } \\
\text { analysis }\end{array}$ & $\begin{array}{l}\text { Teachers felt incompetent to utilise } \\
\text { HoM in teaching because of a lack of } \\
\text { knowledge in HoM, lack of resources } \\
\text { and its exclusion in the syllabus and } \\
\text { examination. They commonly use } \\
\text { HoM by telling the biographies of } \\
\text { Mathematicians instead of focusing } \\
\text { on Mathematics information. }\end{array}$ \\
\hline 25 & $\begin{array}{l}\text { Buchho } \\
\text { Itz \& } \\
\text { Schorch } \\
\text { t (2019) }\end{array}$ & $\begin{array}{l}\text { Germa } \\
\text { ny }\end{array}$ & $\begin{array}{l}\text { Different Facets } \\
\text { of Pre-Service } \\
\text { Teachers' Beliefs } \\
\text { On The History } \\
\text { of Mathematics }\end{array}$ & $\begin{array}{l}141 \text { pre- } \\
\text { service } \\
\text { teachers }\end{array}$ & Questionnaires & $\begin{array}{l}1 . \quad \text { Views } \\
\text { about } \\
\text { HoM } \\
2 . \\
\text { Integratio } \\
\text { n of HoM }\end{array}$ & $\begin{array}{l}\text { 1. } \\
\text { Descriptive } \\
\text { analysis } \\
\text { 2. CFA }\end{array}$ & $\begin{array}{l}\text { Overall, majority of pre-service } \\
\text { teachers support and have positive } \\
\text { beliefs about the usage of HoM in the } \\
\text { classroom. Factors that affect their } \\
\text { beliefs are timing, relevance and } \\
\text { complexity of using HoM in teaching. }\end{array}$ \\
\hline 26 & $\begin{array}{l}\text { Kusum } \\
\text { awati \& } \\
\text { Fachru } \\
\text { din } \\
\text { (2019) }\end{array}$ & $\begin{array}{l}\text { Indone } \\
\text { sia }\end{array}$ & $\begin{array}{l}\text { Analisis Sikap } \\
\text { dan Keyakinan } \\
\text { Calon Guru di } \\
\text { Indonesia } \\
\text { terhadap } \\
\text { Pemanfaatan } \\
\text { Sejarah } \\
\text { Matematika } \\
\text { dalam } \\
\text { Pembelajaran } \\
\text { Matematika }\end{array}$ & $\begin{array}{l}349 \text { teacher } \\
\text { candidates }\end{array}$ & $\begin{array}{l}\text { Attitude and } \\
\text { Belief } \\
\text { Questionnaires }\end{array}$ & $\begin{array}{l}\text { Views } \\
\text { about } \\
\text { HoM }\end{array}$ & $\begin{array}{l}\text { Quantitativ } \\
\text { e } \\
\text { descriptive } \\
\text { analysis }\end{array}$ & $\begin{array}{l}\text { Attitudes and beliefs on the usage of } \\
\text { HoM of teacher candidates are at a } \\
\text { moderate level. Besides, the self- } \\
\text { efficacy scores in using HoM in } \\
\text { teaching and learning are still low. }\end{array}$ \\
\hline 27 & $\begin{array}{l}\text { Bütüne } \\
\text { r \& Baki } \\
(2020)\end{array}$ & Turkey & $\begin{array}{l}\text { The Use of } \\
\text { History of } \\
\text { Mathematics in } \\
\text { the } \\
\text { Mathematics }\end{array}$ & $\begin{array}{l}248^{\text {th }} \text { grade } \\
\text { students }\end{array}$ & $\begin{array}{l}1 . \quad \text { Written } \\
\text { opinion form } \\
2 . \quad \text { Semi- } \\
\text { structured } \\
\text { interviews }\end{array}$ & $\begin{array}{l}\text { Integratio } \\
n \text { of HoM }\end{array}$ & $\begin{array}{l}\text { Action } \\
\text { Study }\end{array}$ & $\begin{array}{l}\text { Students found Mathematics is fun } \\
\text { and interesting after being involved } \\
\text { in activities related to the HoM in } \\
\text { classroom. HoM also helped to }\end{array}$ \\
\hline
\end{tabular}


INTERNATIONAL JOURNAL OF ACADEMIC RESEARCH IN BUSINESS AND SOCIAL SCIENCES Vol. 11, No. 4, 2021, E-ISSN: 2222-6990 @ 2021 HRMARS

\begin{tabular}{|c|c|c|c|c|c|c|c|c|}
\hline & & & $\begin{array}{l}\text { Classroom: An } \\
\text { Action Study }\end{array}$ & & & & & $\begin{array}{l}\text { strengthen their beliefs about } \\
\text { Mathematics. }\end{array}$ \\
\hline 28 & $\begin{array}{l}\text { Kapofu } \\
\& \\
\text { Kapofu } \\
(2020)\end{array}$ & $\begin{array}{l}\text { South } \\
\text { Africa }\end{array}$ & $\begin{array}{l}\text { "This Maths is } \\
\text { better than that } \\
\text { Maths" } \\
\text { Exploring } \\
\text { Learner } \\
\text { Perceptions on } \\
\text { the Integration } \\
\text { of History of } \\
\text { Mathematics in } \\
\text { Teaching the } \\
\text { Theorem of } \\
\text { Pythagoras: A } \\
\text { Case Study }\end{array}$ & $\begin{array}{l}30 \text { grade } \\
11^{\text {th }} \\
\text { students }\end{array}$ & $\begin{array}{l}\text { 1. Group } \\
\text { interviews } \\
2 . \quad \text { Journal } \\
\text { entries }\end{array}$ & $\begin{array}{l}\text { Integratio } \\
\mathrm{n} \text { of HoM }\end{array}$ & Case study & $\begin{array}{l}\text { Integration of HoM in Mathematics } \\
\text { classrooms develops students' } \\
\text { positive perceptions about } \\
\text { Mathematics and increases their } \\
\text { motivation and readiness to learn } \\
\text { Mathematics. }\end{array}$ \\
\hline 29 & $\begin{array}{l}\text { Kusum } \\
\text { awati } \\
\text { et al. } \\
(2020)\end{array}$ & $\begin{array}{l}\text { Indone } \\
\text { sia }\end{array}$ & $\begin{array}{l}\text { History of } \\
\text { Mathematics for } \\
\text { Teaching } \\
\text { Mathematics: } \\
\text { The Case of } \\
\text { Indonesian } \\
\text { Prospective } \\
\text { Teachers' Beliefs } \\
\text { and Attitudes }\end{array}$ & $\begin{array}{l}305 \\
\text { prospective } \\
\text { teachers }\end{array}$ & $\begin{array}{l}\text { Questionnaires } \\
\text { of Attitude and } \\
\text { Belief in using } \\
\text { HoM to teach } \\
\text { Mathematics }\end{array}$ & $\begin{array}{l}\text { Views } \\
\text { about } \\
\text { HoM }\end{array}$ & $\begin{array}{l}\text { Independen } \\
t \text { sample t- } \\
\text { test }\end{array}$ & $\begin{array}{l}\text { Prospective teachers had positive } \\
\text { attitudes and beliefs about using } \\
\text { HoM to teach Mathematics. Those } \\
\text { who had taken the HoM course had a } \\
\text { higher score compared to those who } \\
\text { had not taken the course before. } \\
\text { However, both groups showed low } \\
\text { scores in the questionnaire item on } \\
\text { self-efficacy beliefs. }\end{array}$ \\
\hline 30 & $\begin{array}{l}\text { Li \& He } \\
\text { (2020) }\end{array}$ & China & $\begin{array}{l}\text { Collision } \\
\text { Between } \\
\text { Mathematics } \\
\text { History and High } \\
\text { School Teaching }\end{array}$ & - & $\begin{array}{l}\text { Content } \\
\text { analysis }\end{array}$ & $\begin{array}{l}\text { Integratio } \\
n \text { of HoM }\end{array}$ & $\begin{array}{l}\text { Qualitative } \\
\text { analysis }\end{array}$ & $\begin{array}{l}\text { HoM is beneficial for students by } \\
\text { developing a new learning } \\
\text { environment, increasing students' } \\
\text { enthusiasm to learn Mathematics, } \\
\text { get knowledge about the origin of } \\
\text { Mathematics, encourage students to }\end{array}$ \\
\hline
\end{tabular}


INTERNATIONAL JOURNAL OF ACADEMIC RESEARCH IN BUSINESS AND SOCIAL SCIENCES

Vol. 11, No. 4, 2021, E-ISSN: 2222-6990 @ 2021 HRMARS

\begin{tabular}{|l|l|l|l|l|l|l|}
\hline & $\begin{array}{l}\text { From HPM } \\
\text { Perspective }\end{array}$ & & & $\begin{array}{l}\text { think and construct ideas by } \\
\text { themselves, explore the beauty in } \\
\text { learning Mathematics and allowing } \\
\text { them to fall in love with } \\
\text { Mathematics. }\end{array}$ \\
\hline
\end{tabular}




\section{Discussion}

\section{Mathematics Teachers' Views about the Integration of HoM}

From Table 1, it can be summarized that most of the teachers have positive views about the integration of the History of Mathematics (HoM) (Alpaslan \& Haser, 2012; Alpaslan, Işiksal, \& Haser, 2014; Baki \& Gürsoy, 2018; Burns, 2010; Dejic \& Mihajlovic, 2014; Fadlelmula, 2015; Fraser \& Koop, 1978; Galante, 2014; Kusumawati et al., 2020; Panasuk \& Bolinger Horton, 2011; Buchholtz \& Schorcht, 2019; Tol, 2015; Weldeana \& Abraham, 2014). Three of the articles revealed that most of the teachers agreed that practicing the integration of HoM in their teaching would be beneficial for them as well as the students (Baki \& Gürsoy, 2018; Fadlelmula, 2015; Galante, 2014). Panasuk \& Bolinger-Horton (2011) believed that HoM has its place and values in the school Mathematics curriculum. Two of the articles stated that integrating HoM in the teaching and learning process could help to ensure effective and permanent learning of the students, enhance students' motivation and increase students' interest in learning Mathematics (Panasuk \& Bolinger-Horton, 2011; Tol, 2015). Content knowledge and pedagogical content knowledge possessed by a teacher can be strengthened by applying HoM in teaching. Including HoM in teaching is also seen as one of the best alternative ways or useful strategies to deliver Mathematical knowledge effectively to the students in school (Galante, 2014). One of the articles revealed that teachers who had taken an HoM course in their study before had higher attitude and belief scores towards using HoM in teaching compared to ones who had not taken a course before (Kusumawati et al., 2020).

Kusumawati et al. (2020) in their studies, revealed that teachers' self-efficacy beliefs towards using HoM in their teaching are still low. Two of the studies stated that the teachers did not consider applying the integration of HoM in their teaching although they had positive views about the integration of HoM in teaching (Alpaslan \& Haser, 2012; Fraser \& Koop, 1978). Alpaslan and Haser (2012) in their studies, stated that teachers did not consider using HoM in teaching because they felt that using HoM in Mathematics education is not so important. In addition, using HoM materials in class might lead to wasting time if it is not fully prepared because they require the teachers to possess specific skills to apply HoM materials in their teaching (Fraser \& Koop, 1978).

Despite having positive views about HoM, some of the studies revealed that many teachers encountered problems in applying HoM in class (Alpaslan et al., 2014; Baki \& Gürsoy, 2018; Bașibüyük \& Șahin, 2019; Bütüner, 2017; Tol et al., 2015). Three of the articles mentioned knowledge of HoM as the factor in integrating HoM in teaching (Alpaslan \& Haser, 2012; Bașibüyük \& Șahin, 2019; Bütüner 2017). Bütüner (2017); Bașibüyük and Șahin (2019) in their studies, stated that teachers did not utilise HoM in teaching due to a lack of knowledge on HoM. Bütüner (2017) stated that teachers in general had a low level of knowledge in HoM and those who did not practice HoM had a lower level of knowledge in HoM compared to ones who applied it in teaching. Alpaslan and Haser (2012) revealed that pre-service teachers had moderate knowledge of HoM and their knowledge of HoM increased as their teaching year increased. It was also revealed that the knowledge scores on HoM of male teachers were slightly higher than female teachers.

Another factor that affects teachers' views of applying HoM in teaching is a lack of resources and materials (Baki \& Gürsoy, 2018; Bașibüyük \& Șahin, 2019; Dejic \& Mihajlovic, 2014; Tol, 2015). Buchholtz \& Schorcht (2019) revealed that some pre-service teachers think that HoM is too complex for students to learn. Besides that, three articles mentioned a lack of time as the factor (Baki \& Gürsoy, 2018; Dejic \& Mihajlovic, 2014; Schorcht \& Buchholtz, 2019). One article stated that teachers have no idea where and how to integrate HoM in 
teaching (Fadlelmula, 2015) and three articles emphasised its exclusion in the syllabus and examination as a factor for why teachers did not apply HoM in class (Bașibüyük \& Șahin, 2019; Bütüner, 2017; Schorcht \& Buchholtz, 2019).

\section{Implementation of HoM in Teaching and Learning Mathematics}

Based on Table 1, four of the articles had done the research on the integration of HoM in Mathematics education. Two of the articles revealed that most of the teachers did not implement HoM in their teaching because they felt incompetent to do so due to problems such as having a low level of knowledge in HoM, a lack of resources and materials, a lack of time and exclusion of HoM from the Mathematics syllabus and examination (Bașibüyük \& Șahin, 2019; Dejic \& Mihajlovic, 2014; Bütüner, 2017). One of the articles' findings stated that instead of focusing on Mathematical concepts and information, teachers commonly practice HoM in their teaching by telling the biographies and life stories of Mathematicians (Bașibüyük \& Șahin, 2019). Buchholtz \& Schorcht (2019) in their study also revealed that some pre-service teachers think that HoM it too complex for students to learn. Thus, they rejected the usage of HoM in their teaching.

Besides that, one article discussed the factors influencing the integration of HoM in teaching and learning Mathematics. Factors that influenced teachers' decisions in terms of integrating HoM into their teaching are their beliefs about the importance of HoM, benefits of HoM and values of HoM in the Mathematics curriculum (Panasuk \& Horton, 2013). Panasuk and Horton (2013) listed the factors that might become problems for a teacher in applying HoM in their teaching: (1) level of knowledge of HoM; (2) materials and resources; (3) high stakes testing and (4) enjoyment of teaching and learning HoM. Te Bolinger-Horton \& Panasuk (2011)achers who have a fallibilist perspective about the nature of Mathematics are more likely to integrate HoM in their lessons while teachers who have an absolutist perspective about the nature of Mathematics are less likely to integrate HoM in teaching. Teachers who have an absolutist perspective have a rigid procedure in their teaching process and are less interested in creating new learning opportunities while teachers with a fallibilist perspective are open to changes and development in their teaching style.

Liu (2003) listed five reasons why teachers need to implement HoM in teaching. The first reason is HoM helps to increase students' motivation besides evolving a positive attitude towards learning Mathematics. This is supported by the finding from meta-analysis research done by Bütüner (2015) that stated that using HoM in Mathematics education develops students' positive attitude to learning Mathematics. Integration of HoM in the Mathematics classroom develops students' positive perceptions about Mathematics as well as increasing their motivation and readiness to learn Mathematics (Kapofu \& Kapofu, 2020). Next, HoM also helps to explain what students find difficult now in solving Mathematical problems by referring to the obstacles faced in the history of the development of Mathematics. Besides, HoM helps to develop students' mathematical thinking, reveals the human side of mathematical knowledge and can act as a guide to Mathematics teachers for teaching.

Jankvist (2009) in his research, listed why we need to use HoM in Mathematics education and how to use HoM in Mathematics education. HoM is highly recommended in teaching because: (1) history acts as a tool to assist actual teaching and learning of Mathematics in the classroom and (2) history acts as a goal in the evolution of Mathematics as a subject. HoM can be applied in the teaching and learning process by using three approaches. First, there is the illumination approach. In this approach, historical information is used in teaching. Second, there is the modules approach. In the modules approach, teachers 
can use history-based materials when teaching a small topic. Third is the history-based approach where the idea of teaching is directly inspired by or based on HoM.

HoM may be implemented in Mathematics education in three ways, which are learning history by giving direct information on history, learning Mathematical topics by using a historical-inspired teaching strategy, and developing a deepened awareness of the social and cultural contexts of Mathematics. These are a few examples of tools and ideas on how to integrate HoM in class: (1) historical snippets, (2) history-based projects, (3) primary sources, (4) worksheets, (5) historical packages, (6) discussion on historical issues and arguments, (7) history-related problems, (8) mechanical tools, (9) experiential learning, (10) role playing and games, (11) visual tools, (12) outdoor learning and (13) internet sources (Tzanakis et al., 2002).

Ho (2008) listed the potential, limits and risks of implementing HoM in teaching and learning Mathematics. The possible potentialities of using HoM in the classroom are: (i) understanding the topic much better, ii) presenting different learning situation and (iii) developing positive attitudes of the teachers and students towards teaching and learning Mathematics. However, teachers will face a few limitations in applying HoM in their teaching: (i) a lack of training, (ii) a lack of time and (iii) a lack of rubrics related to HoM. Other than that, implementation of HoM in teaching and learning might involve a few risks such as: (i) teachers being unable to make a relevant connection between HoM and the topic, (ii) it may lead to wasting time if the lesson is not planned carefully, and (iii) students are not familiar with the cultural differences from the past and might wrongly interpret and understand the word 'history' in HoM.

It is found that implementing activities related to HoM in teaching and learning attract students' interests. Li \& He (2020) found that HoM is beneficial for students by developing a new learning environment, increasing students' enthusiasm to learn Mathematics, knowledge on the origin of Mathematics, encouraging students to think and construct ideas by themselves, exploring the beauty in learning Mathematics and letting them fall in love with Mathematics. Students also have the opportunity to discover different methods to solve mathematical problems (Goktepe \& Ozdemir, 2013). In addition, practising activities related to HoM in class can develop a fun and interesting learning experience for the students and deepen their beliefs towards Mathematics ( Bütüner \& Baki, 2020). This is supported by Ozdemir et al. (2012) in their study who have found that HoM helps to strengthen students' geometric proof skills besides attracting their interest in terms of learning the topic. Implementing HoM in the class also has both short-term and long-term effects on students' achievement in Mathematics. Despite this, the implementation of HoM only has short-term positive effects on students' attitudes, anxiety and motivation towards Mathematics (Lim \& Chapman, 2015).

HoM has been integrated in Indonesian Mathematics textbook since 2013 in the form of historical snippets. It is found that this helps students to understand the concepts in Mathematics more deeply. HoM also increases students' motivation and encourages active involvement in the Mathematics' classroom (Ekawati et al., 2018). In Turkey, HoM has also been integrated in Mathematics textbooks. However, most of the HoM elements were only placed in the introduction part of the topics. HoM was also excluded from the content and assessment aspects of the curriculum. It can be concluded that HoM is still not acknowledged as beneficial and not integrated widely in school Mathematics curricula (Şişman, 2018). 


\section{Conclusions}

In conclusion, these systematic review findings have revealed that most of the teachers have positive views, attitudes, and beliefs about using the History of Mathematics (HoM) in teaching and learning Mathematics. Most of them believe that using HoM in Mathematics education is beneficial to them as well as the students. However, the implementation of HoM in teaching is still low due to several problems that need to be addressed by teachers, such as the level of knowledge of HoM, lack of resources and materials, lack of time and exclusion from the syllabus and examination. As for future works, Mendeley, Scopus and Web of Science are highly recommended to be databases of the study using should extensive related keywords.

\section{Acknowledgement}

This research was funded by the grant of Faculty Education, Universiti Kebangsaan Malaysia GG-2019-065.

\section{References}

Alpaslan, M., \& Haser, C. (2012). "History of Mathematics" course for pre-service mathematics teachers: A case study. 12th International Congress on Mathematical Education (July 2012): 4015-4024. http://icme12.org/upload/UpFile2/TSG/0523.pdf.

Alpaslan, M., Işiksal, M., \& Haser, Ç. (2014). Pre-service Mathematics Teachers' Knowledge of History of Mathematics and Their Attitudes and Beliefs Towards Using History of Mathematics in Mathematics Education. Science and Education 23(1): 159-183.

Astin, J., Brown, G., \& Fowler, D. (2016). The Experience of History in Mathematics Education. For the Learning of Mathematics - An International Journal of Mathematics Education 11(2): 7-9.

Bailey, J. (2017). Embedding problem-solving in a primary mathematics programme. Waikato Journal of Education 22(4): 19-31.

Baki, A., \& Gürsoy, K. (2018). Does Using History of Mathematics Make Sense? The Views of Teacher Candidates. Journal of Pedagogical Research 2(2): 78-90. www.ijopr.com.

Baki, A., \& Guven, B. (2009). Khayyam with Cabri: Experiences of pre-service mathematics teachers with Khayyam's solution of cubic equations in dynamic geometry environment. Teaching Mathematics and its Applications 28(1): 1-9.

Bașibüyük, K., \& Șahin, Ö. (2019). Mathematics Teachers' Opinion about the History of Mathematics. Acta Didactica Napocensia 12(2): 117-132.

Bidwell, J. K. (1993). Humanize your classroom with the history of mathematics. The Mathematics Teacher 86(6): 461-464.

Bolinger-Horton, L., Panasuk, R. M. (2011). Raising Awareness the History of Mathematics in High School Curriculum. International Journal of Humanities \& Social Science 1(16): 37. http://connection.ebscohost.com/c/articles/73445106/raising-awareness-historymathematics-high-school-curriculum.

Buchholtz, N., \& Schorcht, S. (2019). Different facets of pre-service teachers' beliefs on the history of mathematics. Proceedings of the Eighth European Summer University on History and Epistemology in Mathematics Education ESU 8 155-175. https://www.researchgate.net/publication/337000343.

Burns, B. (2010). Pre-Service Teachers' Exposure to Using the History of Mathematics to Enhance Their Teaching of High School Mathematics. Issues in the Undergraduate Mathematics Preparation of School Teachers 4(1992) 
Bütüner, S. (2015). Impact of Using History of Mathematics on Students' Mathematics Success: A Meta-Analysis Study. Mevlana International Journal of Education (MIJE) 3(4): 78.

Bütüner, S. Ö. (2017). Secondary School Mathematics Teachers' Knowledge Levels and Use of History of Mathematics. Journal of Education and Training Studies 6(1): 9.

Bütüner, S. Ö., \& Baki, A. (2020). The Use of History of Mathematics in the Mathematics Classroom: An Action Study. International Journal of Education in Mathematics, Science and Technology 8(2): 92.

Charalambous, C. Y., Panaoura, A., \& Philippou, G. (2009). Using the history of mathematics to induce changes in preservice teachers' beliefs and attitudes: Insights from evaluating a teacher education program. Educational Studies in Mathematics 71(2): 161-180.

Cooper, H. (2003). Psychological Bulletin: Editorial. Psychological Bulletin 129(1): 3-9.

Dejic, M., \& Mihajlovic, A. (2014). History of mathematics and teaching mathematics. Inovacije u nastavi 27(3): 15-30.

Ekawati, R., Fiangga, S., \& Siswono, T.Y.E. (2018). Historical aspect of mathematics on Indonesian mathematics textbook. IOP Conference Series: Materials Science and Engineering Vol. 434, hlm. 1-6

Fadlelmula, F. K. (2015). Pre-service teachers' point of views about learning history of mathematics: A case study in Turkey. BSHM Bulletin 30(3): 243-252.

Fauvel, J. (1991). Using history in mathematics education. For the learning of mathematics 11(2): 3-6. http://www.jstor.org/stable/40248010.

Fraser, B. J., \& Koop, A. J. (1978). Teachers' opinions about some teaching material involving history of mathematics. International Journal of Mathematical Education in Science and Technology 9(2): 147-151.

Fried, M. N. (2001). Can mathematics education and history of mathematics coexist? Science and Education 10(4): 391-408.

Galante, D. (2014). The Use of the History of Mathematics in the Teaching of Preservice Teachers. Redimat 3(2): 110-120.

Goktepe, S., \& Ozdemir, A. (2013). An Example of Using History of Mathematics in Classes. European Journal of Science and Mathematics Education 1(3): 125-136.

Gulikers, I., \& Blom, K. (2001). "A historical angle", a survey of recent literature on the use and value of history in geometrical education. Educational Studies in Mathematics 47(2): 223-258.

Hamidah, M., Nabillah, F. M. J., Maslizah, A.W., \& Balkish, O. (2019). Attributes Affecting Students' Ability in Thinking Skills. Jurnal Pendidikan Malaysia 44(2): 84-100.

Hickman, F., \& Kapadia, R. (1983). A history of mathematics course for teachers. International Journal of Mathematical Education in Science and Technology 14(6): 753-761.

Ho, W. (2008). Using history of mathematics in the teaching and learning of mathematics in Singapore. International Conference on Education, Singapore (1): 1-38. http://math.nie.edu.sg/wkho/Research/My publications/Math Education/hom.pdf.

Choon, H. O., \& A/L Chandrasekaran, K. (2015). A Bayesian Network Approach to Identify Factors Affecting Learning of Additional Mathematics. Jurnal Pendidikan Malaysia 40(2): 185-192.

Jankvist, U.T. (2009). A categorization of the "whys" and "hows" of using history in mathematics education. Educational Studies in Mathematics 71(3): 235-261.

Kapofu, L. K., \& Kapofu, W. (2020). "This Maths is better than that Maths" - Exploring Learner Perceptions on the Integration of History of Mathematics in Teaching the Theorem of 
Pythagoras: A Case Study. International Electronic Journal of Mathematics Education 15(3): em0604.

Kusumawati, I. B., \& Fachrudin, A.D. (2019). Analisis Sikap dan Keyakinan Calon Guru di Indonesia terhadap Pemanfaatan Sejarah Matematika dalam Pembelajaran Matematika. Jurnal Riset Pendidikan dan Inovasi Pembelajaran Matematika (JRPIPM) 3(1): 36-43.

Kusumawati, I. B., Fachrudin, A. D., Kohar, A. W., \& Widadah, S. (2020). History of Mathematics for Teaching Mathematics: The Case of Indonesian Prospective Teachers' Beliefs and Attitudes. Universal Journal of Educational Research 8(6): 2305-2314.

$\mathrm{Li}, \mathrm{T} .$, \& He, Y. (2020). Collision Between Mathematics History and High School Teaching From HPM Perspective. European Journal of Research and Reflection in Educational Sciences 8(5): 6-12.

Li, W. L., Osman, K., \& Maat, S. M. (2019). Opsyen sebagai Moderator dalam Hubungan Berstruktur antara Pengetahuan Asas PdP dengan Amalan Pengajaran Guru Matematik Sekolah Rendah (Options as Moderator in the Structural Relationships between Primary Mathematics Teachers' Basic Knowledge of Teaching . Jurnal Pendidikan Malaysia 44(02): 30-40.

Lim, S. Y., \& Chapman, E.(2015). Effects of using history as a tool to teach mathematics on students' attitudes, anxiety, motivation and achievement in grade 11 classrooms. Educational Studies in Mathematics 90(2): 189-212.

Liu, P. (2003). Do Teachers Need to Incorporate the History of Mathematics in Their Teaching? Mathematics Teacher 96(6): 416-421.

Liyanagunawardena, T. R., Adams, A. A., \& Williams, S. A. (2013). MOOCs: A systematic study of the published literature 2008-2012. International Review of Research in Open and Distance Learning 14(3): 202-227.

Marshall, G. L., \& Rich, B. S. (2000). The Role of History in a Mathematics Class. The Mathematics Teacher 93(08): 704-706.

Moher, D., Liberati, A., Tetzlaff, J. A. D. (2009). Preferred Reporting Items for Systematic Reviews and Meta-Analyses: The PRISMA Statement. PLoS Medicine 6(7): 339.

Nachiappan, S., Rengasamy, K., \& Maniam, V. (2016). Analisis pengajaran guru dalam mata pelajaran matematik ke arah aplikasi kognisi dalam kurikulum standard sekolah rendah melalui kaedah hermeneutik. EDUCATUM Journal of Social Sciences 2(1): 21-41.

Ozdemir, A. S., Goktepe, S., \& Kepceoglu, I. (2012). Using Mathematics History to Strengthen Geometric Proof Skills. Procedia - Social and Behavioral Sciences 46: 1177-1181. http://dx.doi.org/10.1016/j.sbspro.2012.05.270.

Panasuk, R. M., \& Bolinger-horton, L. (2011). Integrating History of Mathematics into Curriculum: What are the Chances and Constraints? International Electronic Journal of Mathematics Education 7(1): 3-20.

Panasuk, R. M., \& Horton, B. L. (2013). Integrating History of Mathematics into the Classroom: Was Aristotle Wrong? Journal of Curriculum and Teaching 2(2): 37-46.

Philippou, G. N., \& Christou, C. (1998). The effects of a preparatory mathematics program in changing prospective teachers' attitudes towards mathematics. Educational Studies in Mathematics 35(2): 189-206.

Schorcht, S., \& Buchholtz, N. (2013). Different facets of pre-service teachers' beliefs on the history of mathematics. Proceedings of the Eighth European Summer University on History and Epistemology hlm. 155-175 http://search.ebscohost.com/login.aspx?direct=true\&db=aph\&AN=108710816\&site=e host-live. 
Tan Şişman, G. (2018). History of Mathematics in the Turkish Middle School Mathematics Curriculum and Textbooks. Cukurova University Faculty of Education Journal 47(1): 188215.

Tol, H. Y., Çenberci, S. (Inag) \& Yavuz, A. (2015). Teachers Views Related To Teaching Of Mathematics Course Subjects With Their Historical Developments. European Journal of Education Studies (Special Issue): 58-73.

Tzanakis, C., Arcavi, A., de Sa, C. C., Isoda, M., Lit, C.-K., Niss, M., de Carvalho, J. P., Rodriguez, M., \& Siu, M.-K. (2002). Integrating history of mathematics in the classroom: an analytic survey. DIm. Fauvel, John \& van Maanen, J. (Edt). History in Mathematics Education hlm. 201-240. Dordrech: Springer.

Weldeana, H. N., \& Abraham, S. T. (2014). The effect of an historical perspective on prospective teachers' beliefs in learning mathematics. Journal of Mathematics Teacher Education 17(4): 303-330. 